\title{
Diagnostic Accuracy of DW MRI in Early Detection of Endometrial Carcinoma
}

\author{
RASHA M. KAMAL, M.D.*; NAGAT MANSOUR KHALIFA, M.D.**; AMR KAMAL, M.D.*** and \\ GHADA F.A. HAMMAD, M.D.** \\ The Department of Radiology, Faculty of Medicine, Cairo University*, National Cancer Institute of ${ }^{\text {Cairo*** }}$ and \\ The Department of Surgery, National Cancer Institute of Cairo***, Egypt
}

\begin{abstract}
Introduction: Endometrial carcinoma is the most common gynecological malignancy and the sixth most common neoplasm worldwide. The role of MRI in patients with histologically proven endometrial cancer is to evaluate the depth of myometrial and cervical invasion and detect pelvic lymph node involvement pre-operatively thereby helping to determine the need for lymph node dissection. DW-MRI provides important new information noninvasively. This unique modality is helpful in initial staging of known malignancies, differentiating benign from malignant lesions, as a biomarker for treatment response and determining the presence of disease recurrence.
\end{abstract}

Aim of Study: The aim of this study is to assess the diagnostic accuracy of diffusion weighted imaging and ADC value in early diagnosis of endometrial cancer in high risk patients decreasing the need for surgical interventions for diagnosis.

Patients and Methods: This study included 33 patients, 21 patients had pathologically proven endometrial cancer, and 12 patients had endometrial hyperplasia with control group of 36 patients referred to the Radiology Department from Surgical Department to assess the endometrium. Pelvic DCE-MR was done and DWI was obtained with $3 \mathrm{~b}$-values including 0,300 and $600 \mathrm{~mm} / \mathrm{sec}$.

Results: There is statistically significant difference between the benign and malignant group with $p$-value $=0.001$ in which Sensitivity $=90.0 \%$ Specificity $=83.3 \%$ Accuracy $=85.2 \%$. The $\mathrm{ADC}$ value of benign group ranged from (0.8 to 2), the ADC value of malignant group ranged from (0.5 to 1.3). There was significant overlap between benign and malignant group as regards the ADC value however if we use cut off value of 0.6 for malignant lesions and 1.6 for benign lesions, the specificity will be $77.1 \%$ for benign and $100 \%$ for hyperplasia. Our results revealed that the inclusion of CE MRI resulted in statistically insignificant improvement of the diagnostic accuracy and sensitivity in differentiation between benign and malignant group $(p 0.06)$ in which Sensitivity $=30.4 \%$ Specificity $=38.5 \%$ Accuracy $=33.3 \%$.

Conclusion: Our results revealed that the inclusion of $\mathrm{CE}$ MRI resulted in statistically insignificant improvement of the

Correspondence to: Dr. Rasha M. Kamal,

The Department of Radiology, Faculty of Medicine, Egypt diagnostic accuracy and sensitivity in differentiation between benign and malignant group. The diffusion weighted imaging ADC value improve the efficacy and diagnostic accuracy in early diagnosis of endometrial cancer in high risk patients decrease the need for surgical interventions for diagnosis.

Key Words: DW MRI-ADC value - Early diagnosis of endometrial cancer.

\section{Introduction}

ENDOMETRIAL carcinoma is the most common gynecological malignancy and the sixth most common neoplasm worldwide. It typically presents with abnormal uterine bleeding in $75 \%$ to $90 \%$ of patients [1]. Uterine carcinoma is usually staged and managed on the basis of criteria proposed by the International Federation of Gynecology and Obstetrics (FIGO). However, the FIGO staging system is sometimes inaccurate, in spite of the fact that accurate staging is essential for appropriate treatment planning [2]. The role of MRI in patients with histologically proven endometrial cancer is to evaluate the depth of myometrial and cervical invasion and detect pelvic lymph node involvement preoperatively thereby helping to determine the need for lymph node dissection [3]. Endometrial hyperplasia is a precursor to the most common endometrioid adenocarcinoma which virtually always results from chronic estrogen stimulation unopposed by the counterbalancing effects of progesterone. It is characterized by a proliferation of endometrial glands resulting in a greater gland to stroma ratio than observed in normal endometrium. It may progress to or coexist with endometrial carcinoma [4] . Functional imaging by means of Dynamic Contrast-Enhanced Magnetic Resonance Imaging (DCE-MRI) and Diffusion Weighted Magnetic Resonance Imaging (DW-MRI) is now part of the standard imaging protocols for evaluation of the female pelvis [5]. DCE-MRI improves the 
accuracy of staging of endometrial cancer and is highly accurate in evaluating the depth of myometrial invasion as majority of tumors are hypo vascular compared to the adjacent enhancing myometrium [5]. Diffusion-Weighted Magnetic Resonance Imaging (DW-MRI) is a functional imaging technique whose contrast derives from the random motion of water molecules within tissues, thus no exogenous contrast medium administration is required, so that diffusion-weighted sequences can now be included in routine patient assessment (Whittaker et al., 2009 [5]). DW-MRI provides important new information noninvasively. This unique modality is helpful in initial staging of known malignancies, differentiating benign from malignant lesions, as a biomarker for treatment response and determining the presence of disease recurrence [6]. DW-MRI can be helpful in cases of tumors that are either iso-or hyperintense relative to the myometrium or when the use of intravenous contrast medium is contraindicated [5]. Both DCEMRI and DW-MRI enable the radiologist to move from morphological to functional assessment of diseases of the female pelvis [5].

\section{Patients and Methods}

This was a prospective study included 33 female patients referred from the Gynecological Outpatient Clinic in NCI Cairo University to Radiological Department to assess the endometrium starting from March 2018 to January 2019. The study was approved by its Research and Ethical Committee with informed patients consent. The patients' age ranged between 30 to 77 years old. The mean age was 59.7 and the median was 60 years old. They present with abnormal uterine bleeding, postmenopausal bleeding and/or vaginal discharge or during routine gynecological follow-up for high risk patient (i.e. under hormonal treatment) as in (Table 1).

Table (1):

\begin{tabular}{lccc}
\hline & $\begin{array}{c}\text { Abnormal } \\
\text { uterine } \\
\text { bleeding }\end{array}$ & $\begin{array}{c}\text { Post-menopausal } \\
\text { bleeding or } \\
\text { vaginal discharge }\end{array}$ & $\begin{array}{c}\text { During routine } \\
\text { gynecological } \\
\text { follow-up for } \\
\text { high risk patient }\end{array}$ \\
\hline Clinical presentation & 4 & 29 & 3 \\
\hline
\end{tabular}

Inclusion criteria: Patients with dysfunctional uterine bleeding, post-menopausal bleeding and patients under tamoxifen were enrolled in our study.

Control group: (36 female patients) their age ranges from 22 to 83 years (mean age 48.6 years) asymptomatic patients performing the MRI study for another purpose.

Prior to staging imaging all cases were subjected to the following: Full history taking with a special emphasis on:

- Parity.

- Age of menarche.

- Duration of menopause

- History of replacement hormonal therapy, contraceptive therapy.

- Hormonal treatment for breast cancer.

- Previous gynecological problem or pervious curettage.

- History of systemic disease or anticoagulant therapy.

MR imaging was performed on a 1.5-T MR Imaging Unit in Radiology Department in National Cancer Institute Cairo University (Achieva, Philips medical system). All the patients were imaged in the supine position using pelvic phased-array Torso coil.

MR imaging protocol: Pre-contrast Sequences Survey Sagittal T2; FOV (FH=300mm, RL $=150$ $\mathrm{mm}, \mathrm{AP}=300 \mathrm{~mm}$ ) Scan Plane: Oblique.Coronal T2; FOV $(\mathrm{FH}=300 \mathrm{~mm}, \mathrm{RL}=300 \mathrm{~mm}, \mathrm{AP}=150 \mathrm{~mm})$. Axial T2; Scan plane: $\mathrm{FOV}(\mathrm{FH}=211, \mathrm{AP}=250 \mathrm{~mm}$, $\mathrm{RL}=274 \mathrm{~mm}$ ) Scan plane: Oblique. Axial T1; FOV: $(\mathrm{AP}=250 \mathrm{~mm}, \mathrm{RL}=274 \mathrm{~mm}, \mathrm{FH}=211 \mathrm{~mm})$ Scan Plane: Oblique FOV $9 \mathrm{AP}=320 \mathrm{~mm}, \mathrm{RL}=260 \mathrm{~mm}$, $\mathrm{FH}=200 \mathrm{~mm})$. Axial DWI 3 b-values $(0 / 300 / 600)$ Slice thickness: $7 \mathrm{~mm}$ Slice Spacing: $1 \mathrm{~mm}$. post contrast sequences e-Thrive (T1 high resolution isotropic volume excitation fast gradient, $3 \mathrm{D}, \&$ Fat-sat) FOV: $(\mathrm{AP}=271 \mathrm{~mm}, \mathrm{RL}=255 \mathrm{~mm}, \mathrm{FH}=$ $252 \mathrm{~mm}$ ) Slice thickness: $3 \mathrm{~mm}$. 3D thickness $=3$ Slice gap: $0 \mathrm{~mm}$ Number of slices $=84$ Scan Plane: Sagittal.

Image interpretation: MR images were analyzed for the following parameters: The morphological MRI features was independently reviewed by 2 experienced radiologists including:

a- Tumor signal intensity on T1, T2-weighted image compared with that of adjacent myometrium.

b- Thickness of the endometrium.

c- Endometrial enhancement pattern in post contrast images: homogenous or heterogeneous/faint, moderate or intense.

d- Myometrial infiltration. 
e- Tumor size on T2-weighted images and post contrast images.

f- Uterine, vaginal, parametrial, upward and downward extension, T2-weighted images, post contrast images and DWI.

g- Ureteric and urinary bladder invasion, rectal invasion (i) Presence of enlarged pelvic and/or para-aortic lymph nodes (cut off value, $10 \mathrm{~mm}$ along the minimal transverse diameter).

ADC calculation: Regarding the quantitative analysis of DWI, we generated the ADC map, and then we selected the ROI manually. The ADC value was automatically calculated on the work station to get mean ADC value \& MRDA (least ADC value/maximum restricted diffusion; MRDA) (X $10^{-3} \mathrm{~mm} / \mathrm{s}$ ). To ensure that the same areas were measured, regions of interest were copied and pasted from DW images to ADC maps. Data were transferred at a workstation (Extended Workspace Philips, Philips Medical Systems, Best, and The Netherlands).

For the qualitative analysis; the signal intensity of the lesions on DWI (b1000s $/ \mathrm{mm} 2$ ) was evaluated visually. The lesion was isointense-hypointense if it had a signal intensity equal or lower to the myometrium. The lesion was hyperintense if it had a signal intensity higher to the myometrium. While comparing patients within the same group, lesions characterized by "hyperintense" signal are classified in one subgroup, and the lesions which are characterized by "isointense or hypointense" signal were gathered in another subgroup.

For the quantitative analysis, we placed Regions of Interest (ROIs) measuring at least $0.02 \mathrm{~cm} 2$ on the lesions on DWI (b $1000 \mathrm{~s} / \mathrm{mm}^{2}$ ), ADC maps, and postcontrast images (delayed phases) avoiding obvious areas of inhomogeneity and artifacts. We also avoid the junctional zone of the uterus, the cystic-necrotic components of the lesions, and the peduncle of the polyps.

We performed our measurement from the enhancing components in cases where contrast material was administered and also from homogenous areas and areas of the lesion close to the fundus, while avoiding inhomogeneous areas. In addition, we avoid the hemorrhagic areas. The ROI was placed within the lesions in the area with the lowest $\mathrm{ADC}$ value on ADC Map and highest intensity on DWI (b1000s/mm2). In post contrast images, the ROI has been placed to the areas with the most enhancement. At least three measurements were performed and averaged for each lesion.
For a comparative analysis of the DWI (b1000 $\mathrm{s} / \mathrm{mm}^{2}$ ) and contrast enhancement pattern, we divided the average intensity within the lesions by the intensity of the myometrium in the DWI and post contrast images $(\mathrm{b} 1000 \mathrm{q}=\mathrm{DWI}$ signal-intensity lesion/DWI signal-intensity myometrium, $\mathrm{Cq}=$ post contrast signal-intensity lesion/post contrast signal-intensity myometrium).

\section{Results}

\section{Contrast enhanced MRI imaging features:}

Endometrial thickness: All patients (36/36, $100 \%)$ in the control group showed average endometrial thickness. All hyperplasia (12/12, 100\%) and carcinoma $(21 / 21,100 \%)$ groups showed a thickened endometrial lining (more than $6 \mathrm{~mm}$ in post menopausal and persistent thickening in premenopausal) there was significant difference between the endometrial thickness of the control group as compared to hyperplasia and carcinoma groups ( $p$-value 0.001 ).

Endometrium T2 signal: (34/36, 94.4\%) of the control group showed normal high T2 signal. (3/12, $25 \%$ ) of the hyperplasia group patients showed high T2 signal of the endometrium while $(9 / 12$, $75 \%)$ showed low T2 signal. (20/21, 95.2\%) of the carcinoma group showed low T2 signal there was no significant difference between the signal pattern of the carcinoma group as compared to the control and hyperplasia group ( $p$-value 0.87 ).

Myometrial invasion: All control group (36/36, $100 \%)$ and hyperplasia group $(12 / 12,100 \%)$ showed intact junctional zone with no myometrial invasion. $(17 / 21,80.9 \%)$ of the carcinoma group showed disrupted junctional zone with myometrial invasion. There was significant difference between the control and hyperplasia group as compared to the carcinoma group as regards the myometrial invasion| ( $p$-value 0.001).

Dynamic contrast enhanced MRI: All control group $(36 / 36,100 \%)$ showed normal enhancement timing. (7/12, 58.3\%) of the Hyperplasia group showed delayed enhancement. $(16 / 21,76 \%)$ of Carcinoma group showed delayed enhancement. There was no significant difference between the delayed contrast enhancement of the carcinoma group as compared to the control and hyperplasia group ( $p$-value 0.06 ).

Diagnostic indices of contrast enhanced MRI contrast enhanced MRI showed low sensitivity and specificity in differentiation between hyperplasia and carcinoma groups (Table 2). 
Table (2): Diagnostic indices of contrast enhanced MRI.

\begin{tabular}{ll}
\hline TP (True Positive) & 14 \\
FP (False Positive) & 7 \\
TN (True Negative) & 7 \\
FN (False Negative) & 5 \\
Sensitivity & $30.4 \%$ \\
Specificity & $38.5 \%$ \\
PPV (Positive Predictive Value) & $46.7 \%$ \\
NPV (Negative Predictive Value) & $23.8 \%$ \\
Accuracy & $33.3 \%$ \\
\hline
\end{tabular}

Diffusion sequence: Diffusion weighted images: $(34 / 36,94.4 \%)$ of the control group showed normal facilitated diffusion of endometrium. (8/12, 66.6\%) of the hyperplasia group showed normal facilitated diffusion of endometrium. (20/21, 95.2\%) of the carcinoma group showed restricted diffusion of endometrium. There was significant difference between the control and hyperplasia group as compared to the carcinoma group as regards the pattern of diffusion weighted images $\mid$ ( $p$-value $0.001)$.

Diagnostic indices of DWI showed high sensitivity and specificity in differentiation between hyperplasia (Table 3).

Table (3): Diagnostic indices of DWI.

\begin{tabular}{ll}
\hline TP (True Positive) & 20 \\
FP (False Positive) & 4 \\
TN (True Negative) & 8 \\
FN (False Negative) & 1 \\
Sensitivity & $90 \%$ \\
Specificity & $83.3 \%$ \\
PPV (Positive Predictive Value) & $69.2 \%$ \\
NPV (Negative Predictive Value) & $95.2 \%$ \\
Accuracy & $85.2 \%$ \\
\hline
\end{tabular}

When adding the results of CE-MRI to DWI the diagnostic accuracy in differentiation between the hyperplasia and carcinoma group decreased to $80 \%$ as compared to $85.2 \%$ in case of DWI alone (Table 4).

Table (4): Added value of CE-MRI and DWI.

\begin{tabular}{lclc}
\hline & CE-MRI & DWI & Added value of both \\
\hline Sensitivity & $30.4 \%$ & $90 \%$ & $76.9 \%$ \\
Specificity & $38.5 \%$ & $83.3 \%$ & $81.8 \%$ \\
PPV & $46.7 \%$ & $69.2 \%$ & $71.4 \%$ \\
NPV & $23.8 \%$ & $95.2 \%$ & $85.7 \%$ \\
Accuracy & $33.3 \%$ & $85.2 \%$ & $80 \%$ \\
\hline
\end{tabular}

$A D C$ value and detailed analysis of $A D C$ values of the cases and control groups: As regards the cases: The average ADC values ranged from 0.5 to 2 Fig. (1) the mean ADC values for the benign group was 1.3 with $\mathrm{SD} \pm 0.4$ while malignant group was 0.9 with $\mathrm{SD} \pm 0.1$ when comparing both groups there was statistically significant difference $(p$ value 0.001$)$ with overlap ranged from ( 0.8 to 1.3$)$ Fig. (2).

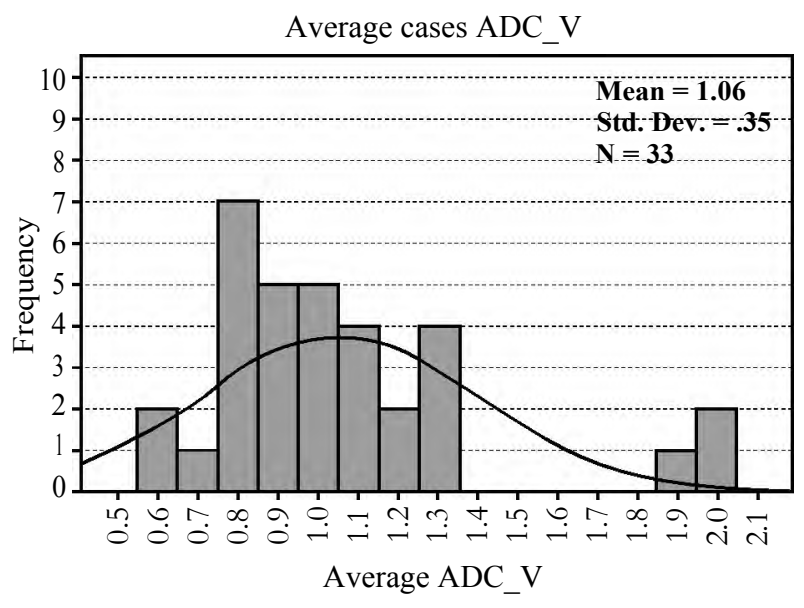

Fig. (1): The average ADC value of the cases.

There is statistically significant difference between ADC value of the hyperplasia and carcinoma group $(p$-value $=0.001)($ Table 5$)$.

Table (5): $p$-value of average ADC of cases.

\begin{tabular}{lcccc}
\hline & $\mathrm{N}$ & Mean & Std. Deviation & $p$-value \\
\hline Hyperplasia & 12 & 1.333 & 0.4141 & $=0.001$ \\
Carcinoma & 21 & 0.900 & 0.1732 & \\
\hline
\end{tabular}

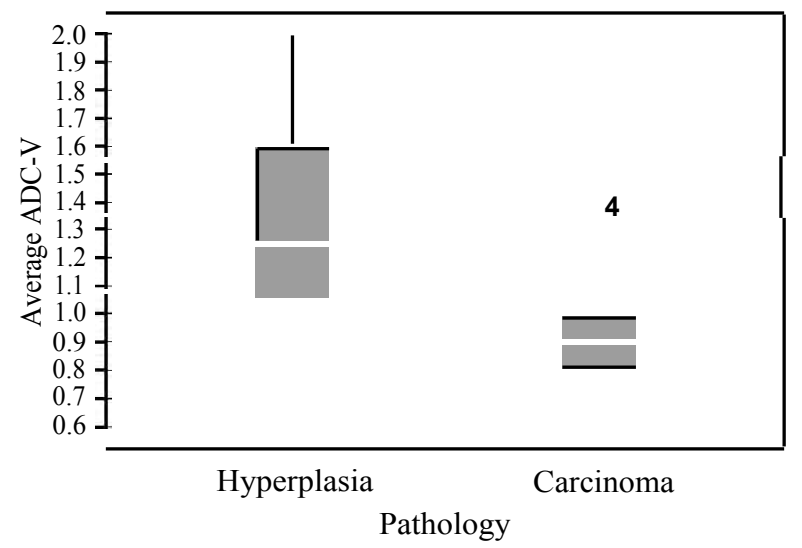

Fig. (2): Comparison of ADC value of hyperplasia versus carcinoma groups.

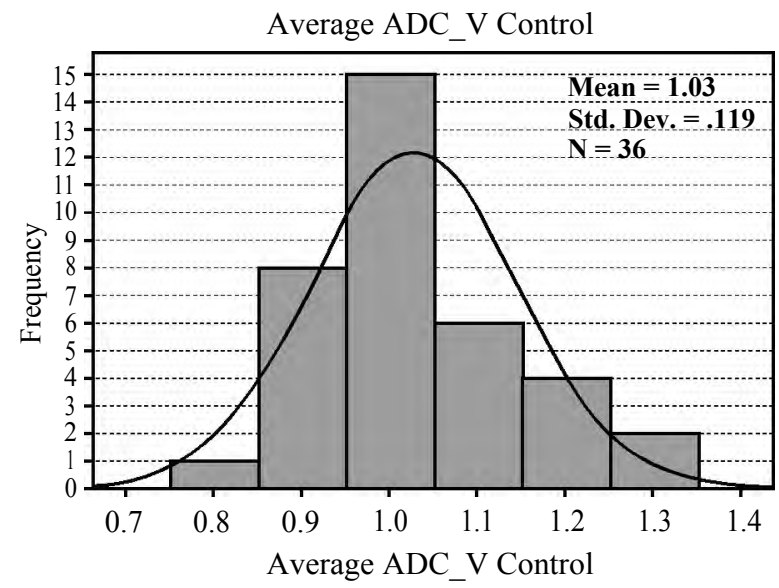

Fig. (3): The average ADC values of the control group. 
Table (6): $p$-value of average ADC of cases versus control.

\begin{tabular}{lcccc}
\hline Average ADC-V & $\mathrm{N}$ & Mean & Std. Deviation & $p$-value \\
\hline Cases & 33 & 1.058 & 0.3500 & $=0.6$ \\
Control & 36 & 1.028 & 0.1186 & \\
\hline
\end{tabular}

Table (7): Conclusion of ADC values.

\begin{tabular}{lccc}
\hline & Hyperplasia & Carcinoma & Control \\
\hline $\mathrm{N}$ & 12 & 21 & 36 \\
Mean & 1.3 & 0.9 & 1.0 \\
Median & 1.2 & 0.9 & 1.0 \\
Minimum & 0.8 & 0.6 & 0.8 \\
Maximum & 2.0 & 1.3 & 1.3 \\
Range & 1.2 & 0.7 & 0.5 \\
\hline
\end{tabular}

Table (8): Cut off value for hyperplasia and carcinoma group.

\begin{tabular}{lcccl}
\hline Parameter & Cutoff value & Sensitivity & Specificity & $p$-value \\
\hline Hyperplasia & $\begin{array}{c}\text { = or more } \\
\text { than 1.6 }\end{array}$ & $52.4 \%$ & $100 \%$ & $\begin{array}{l}\text { Less than } \\
0.001\end{array}$ \\
Carcinoma & $\begin{array}{c}\text { or less } \\
\text { than } 0.6\end{array}$ & $69.9 \%$ & $77.1 \%$ & $\begin{array}{l}\text { Less than } \\
0.001\end{array}$ \\
\hline
\end{tabular}

As regards the control group: The average ADC values ranged from 0.8 to $1.3 \mathrm{Fig}$. (3) the mean $\mathrm{ADC}$ values for the control group was 1.02 with $\mathrm{SD} \pm 0.11$ while cases 1.05 with SD \pm 0.35 (Table $5)$ when comparing both groups there was no statistically significant difference ( $p$-value 0.6 ) with significant overlap.
There is no statistically significant difference ADC value of control and cases $(p$-value $=0.6$ ) (Table 6).

The ADC value of benign lesion ranged from (0.8 to 2), malignant group ranged from ( 0.5 to $1.3)$ and that for control ranged from (0.8 to 1.3$)$ there was significant overlap between lesions as regards the ADC values (Table 7), Fig. (4) however cut off values of $=$ or more than 1.6 for hyperplasia group showed specificity $100 \%$ and that for carcinoma group values $=$ or less than 0.6 showed specificity $77 \%$ (Table 8 ).

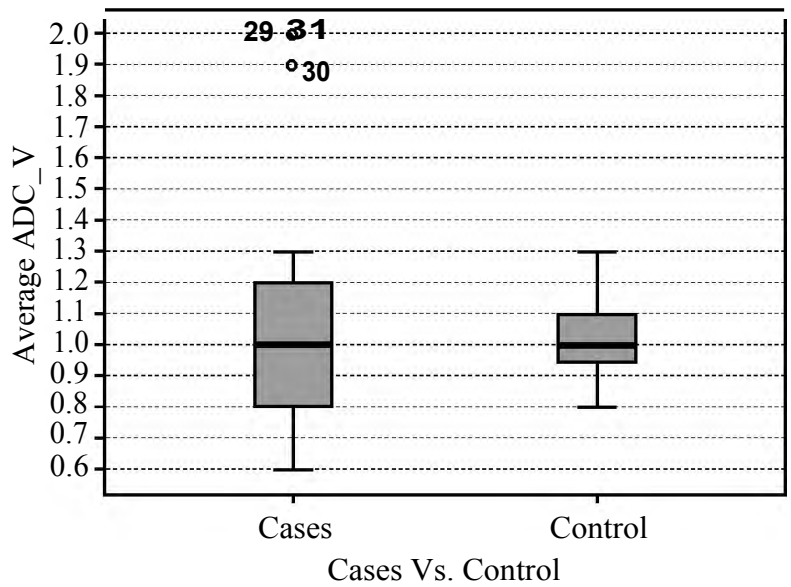

Fig. (4): The overlap between the average ADC values of the cases versus the control group.
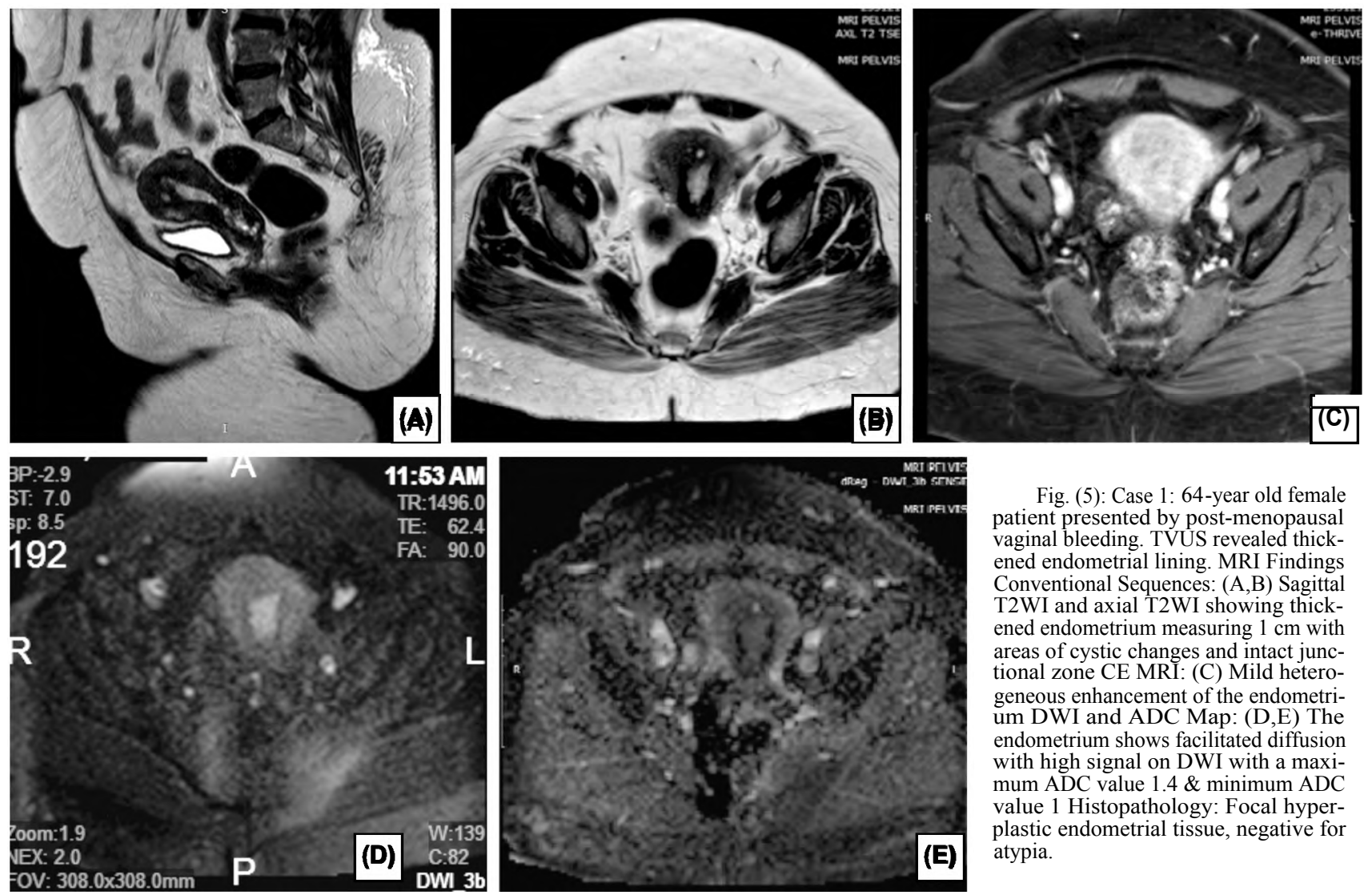

Fig. (5): Case 1: 64-year old female patient presented by post-menopausal vaginal bleeding. TVUS revealed thickened endometrial lining. MRI Findings Conventional Sequences: $(A, B)$ Sagittal T2WI and axial T2WI showing thickened endometrium measuring $1 \mathrm{~cm}$ with areas of cystic changes and intact junctional zone CE MRI: (C) Mild heterogeneous enhancement of the endometrium DWI and ADC Map: (D,E) The endometrium shows facilitated diffusion with high signal on DWI with a maximum ADC value $1.4 \&$ minimum ADC value 1 Histopathology: Focal hyperplastic endometrial tissue, negative for atypia. 

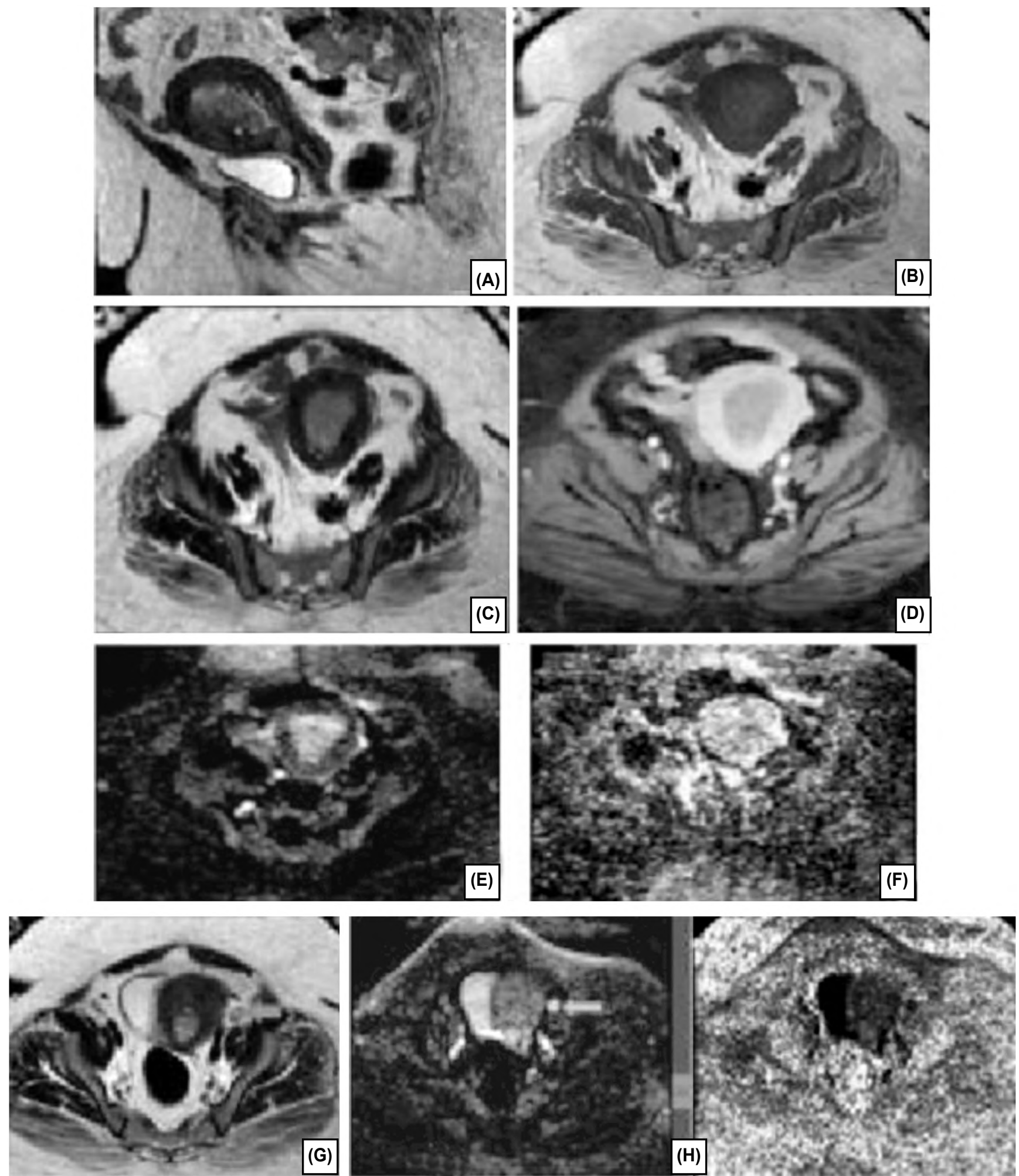

Fig. (6): Case 2: 2:57-year old female patient presented by postmenopausal vaginal bleeding. TVUS revealed thickened endometrial lining. MRI findings: $(\mathrm{A}, \mathrm{B}, \mathrm{C})$ The uterine cavity is seen distended by an ill-defined irregular intra luminal endometrial mass measuring about $1.1 \mathrm{~cm}$ eliciting heterogeneous high T2 signal intensity and iso-intense signal on T1WI with disrupted junctional zone along the left lateral wall of the uterine corpus invading less than $50 \%$ of the myometrial thickness. No pelvic lymphadenopathy. The anterior cervical lip show a rather defined rounded lesion with iso-intense T1 signal and high T2 signal intensity measuring $1.7 \mathrm{~cm}$ in diameter. CE MRI (D) No significant enhancement of the endometrial mass. DWI and ADC Map: (E,F,G,H) The endometrial lesion show high signal on DWI and low signal on the corresponding ADC maps. ADC value was (Max $1.1 \times 10 \mathrm{~mm} / \mathrm{s}$, Minimum $0.78 \times 10^{-3} \mathrm{~mm} / \mathrm{s}$ ). The diffusion WI images showed restricted diffusion of the left ovary raising the possibility of tumoral deposits within it; upstaging the tumoral stage from IA to IIIA. (arrow). (G) Axial T2WI and (H) DWI \& ADC map. Radiological staging: Stage III a Histopathology: Pan hysterectomy: Endometrial adenocarcinoma, Endometrioid type Grade 2. Infiltrating $1 / 4$ the myometrial thickness and positive tumor deposits on the left ovary. Anterior cervical interstitial myoma. 

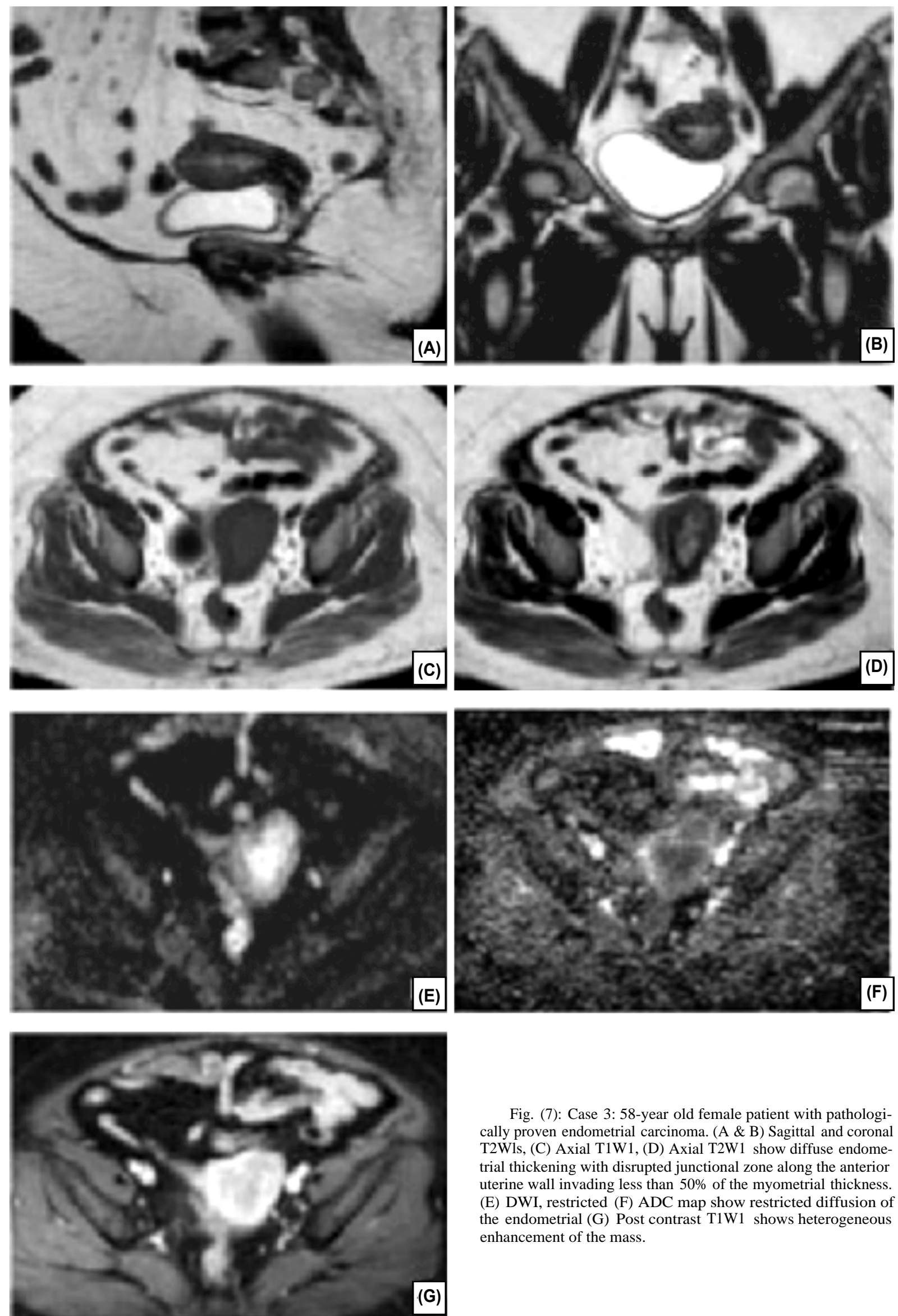

Fig. (7): Case 3: 58-year old female patient with pathologically proven endometrial carcinoma. (A \& B) Sagittal and coronal T2Wls, (C) Axial T1W1, (D) Axial T2W1 show diffuse endometrial thickening with disrupted junctional zone along the anterior uterine wall invading less than $50 \%$ of the myometrial thickness. (E) DWI, restricted (F) ADC map show restricted diffusion of the endometrial (G) Post contrast T1W1 shows heterogeneous enhancement of the mass. 


\section{Discussion}

Trans-vaginal sonography is the procedure of choice for initial evaluation of patients with suspected endometrial carcinoma, the choice of patients for sampling in women with increased endometrial thickness is important. An endometrial thickness of more than $6 \mathrm{~mm}$ at trans-vaginal sonography in patients with postmenopausal bleeding requires endometrial sampling for diagnosis of endometrial cancer. However, if trans-vaginal sonography cannot be performed or if the histopathologic findings are inconclusive, MR imaging can be performed for lesion detection and for staging [7].

Very early-stage small cancers that do not cause significant endometrial thickening may not be detected at conventional MR imaging. However, endometrial cancer shows evidence of restricted diffusion, and DW imaging may be useful in such isolated cases. The normal proliferative endometrium is hypercellular and may demonstrate bright signal intensity on source DW images; however, the ADC values of endometrial cancer are significantly lower than those of the normal endometrium Hence, DW imaging may be used to help differentiate endometrial cancer from the normal endometrium. The ADC values of higher-grade endometrial cancers tend to decrease compared with those of lower-grade cancers. However, the use of DW imaging to differentiate histologic grades of endometrial cancer is much debated because of the considerable overlap reported in ADC values [8].

In this study, we aim to evaluate the efficacy and the added value of the DWI and contrast enhanced MRI in conjunct to conventional pelvic MRI sequences in detection and proper staging of uterine endometrial carcinoma.

The current study included 33 patients, their age ranges from 30 to 70 years old. The mean age was 59.7 years old. Twenty one cases pathologically were proven as endometrial cancer and 12 cases pathologically proven as hyperplasia with control group of 36 patients of normal endometrium. The additions of DWI sequence on routine conventional MR examination contribute to the differential diagnosis of endometrial hyperplasia with endometrial cancer. There is statistically significant difference between the benign and malignant group with $p$-value was 0.001 in which sensitivity was $90.0 \%$ specificity was $83.3 \%$ and accuracy was $85.2 \%$. False positive results were due to secretory changes of the endometrium. False negative were found in few cases of adenocarcinoma, and endometroid carcinoma.

In comparing the results of contrast images to DWI there was no increase in the accuracy of the differentiation between benign ad malignant groups.

In our study, we have demonstrated that the ADC value of benign group ranged from ( 0.8 to 2 ) and for malignant ranged from (0.5 to 1.3$)$ there was significant overlap between both benign and malignant group however if we use a cut off value of 0.6 for malignant lesion and 1.6 for benign lesions respectively will be $77.1 \%$ specificity for malignant and $100 \%$ specificity for benign.

Fujii et al., and Jianq et al., [9,10] have reported that the ADC values differed significantly between malignant $(0.98 \pm 0.19)$ and benign lesions $(1.44 \pm$ $0.34)(p<.01)$.

In a study done by Takeuchi et al., 2009 [11] Published the ADC values in endometrial cancer and benign lesions as $0.84 \pm 0.19$ and $1.58 \pm 0.36$, respectively $(p<.01)$ in our study, the mean ADC values were nearly similar to the other studies results.

The clinical value of our study is in assisting clinician in providing an adequate level of suspicion for endometrial malignancy using DWI properties, on the abnormality detected in the uterine cavity, and when endometrial sampling is inadequate, DWI findings can be helpful for the patients management, DWI properties may allow more confidence and reliability for the diagnosis of benign or malignant endometrial processes compared to conventional MR or transvaginal ultrasound findings.

\section{Conclusion:}

The use of contrast enhanced MR imaging does not improve the accurate differentiation. The use of quantitative DW imaging provide added value in differentiating benign from malignant group sensitivity \& Specificity $90 \% \& 83.3 \%$ respective$1 y$. The ADC value of benign group ranged from (0.8 to 2). The ADC value of malignant group ranged from ( 0.5 to 1.3 ). There was significant overlap between benign and malignant group as regards the ADC value however if we use cut off value of 0.6 for malignant lesions and 1.6 for benign lesions specificity will be $77.1 \%$ for benign and $100 \%$ for hyperplasia. Figs. (5-7). Illustrated cases $1,2,3$. 


\section{References}

1- JEMAL A., BRAY F., CENTER M.M., FERLAY J., WARD E. and FORMAN D.: Global cancer statistics. CA: A Cancer Journal for Clinicians, 61 (2): 69-90, 2011.

2- HORI M., KIM T., MURAKAMI T., et al.: Uterine cervical carcinoma: Pre-operative staging with 3.0-T MR imagingcomparison with 1.5-T MR imaging. Radiology, 251 (1): 96-104, 2009.

3- PEUNGJESADA S., BHOSALE P.R., BALACHAN DRAN A. and IYER R.B.: Magnetic resonance imaging of endometrial carcinoma. Journal of computer assisted tomography, 33 (4): 601-8, 2009.

4- KURMAN R. and NORRIS H.: Endometrial Hyperplasia and Related Cellular Changes. In: Kurman R, ed. Blaustein's Pathology of the Female Genital Tract. New York: Springer, 11: 411-37, 1994.

5- SALA E., ROCKALL A.G., FREEMAN S.J., MITCHELL D.G. and REINHOLD C.: The added role of MR imaging in treatment stratification of patients with gynecologic malignancies: What the radiologist needs to know. Radiology, 266 (3): 717-40, 2013.

6- WHITTAKER C.S., COADY A., CULVER L., RUSTIN G., PADWICK M. and PADHANI A.R.: Diffusionweighted MR imaging of female pelvic tumors: A pictorial review. Radiographics, 29 (3): 759-74, 2009.
7- NOUGARET S., TIRUMANI S.H., ADDLEY H., PANDEY H., SALA E. and REINHOLDO C.: Pearls and pitfalls in MRI of gynecologic malignancy with diffusionweighted technique. AJR. American journal of roentgenology, 200 (2): 261-76, 2013.

8- RECHICHI G.1, GALIMBERTI S., SIGNORELLI M., FRANZESI C.T., PEREGO P., VALSECCHI M.G. and SIRONI S.: Endometrial cancer: Correlation of apparent diffusion coefficient with tumor grade, depth of myometrial invasion, and presence of lymph node metastases AJR Am. J. Roentgenol., 197 (1): 256-62, 2011.

9- FUJII S.1, MATSUSUE E., KIGAWA J., SATO S., KANASAKI Y., NAKANISHI J., SUGIHARA S., KAMINOU T., TERAKAWA N. and OGAWA T.: Diagnostic accuracy of the apparent diffusion coefficient in differentiating benign from malignant uterine endometrial cavity lesions: Initial results. Eur. Radiol., 18 (2): 384-9, 2008.

10- JIANQ J.X., ZHAO J.L., ZHANG Q., QING J.F., ZHNAGS.Q. and ZHANG Y.M.: Endometrial carcinoma: Diffusion-weighted imaging diagnostic accuracy and correlation with KI-67 expression: Clin. Radiol., 73 (4): 413.e1-413.e6, 2018.

11- TAKEUCHI M., MATSUZAKI K. and NISHITANI H.: Diffusion weighted magnetic resonance imaging of endometrial cancer: Differentiation from benign endometrial lesions and pre-operative assessment of myometrial invasion Acta Radiol., 50 (80: 947-53), 2009.

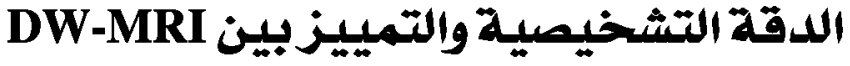

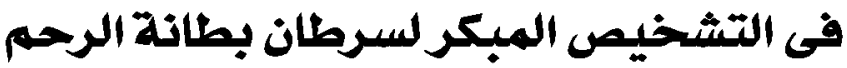

مقدمة: سرطان بطانة الرحم هو الورم الخبيث الآكثر شيوعاً فى آمراض النساء والسادس الآكثر شيوعاً الآتوام فى جميع آنحاء العالم.

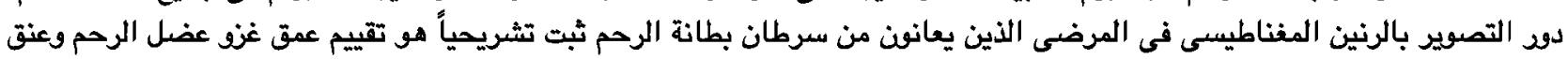

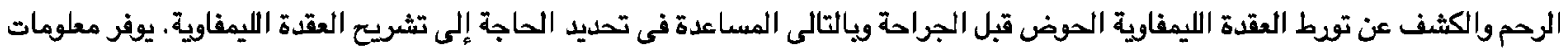

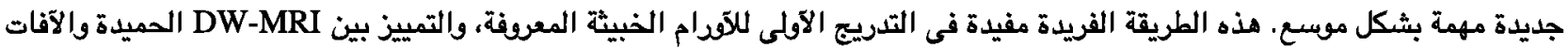

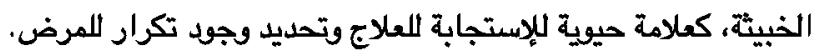

الهدف من هذه الدراسة هو تقييم الدقة التشخيصية للتصوير المونفن النشر وقيمة ADC في التشخيص المبكر لسرطان بطانة الرحم لدى المرضى المعرضين لمخاطر عالية مما يقلل من الحاجة إلى التذخلات الجراحية اللتشيص.

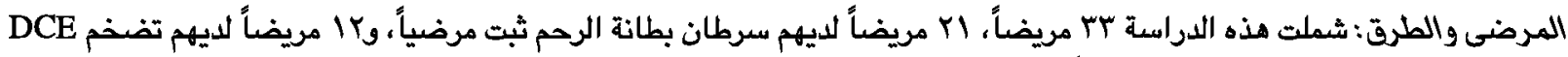

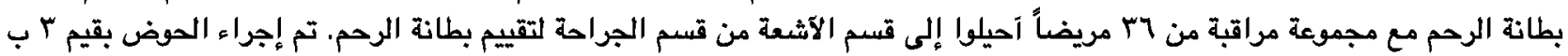

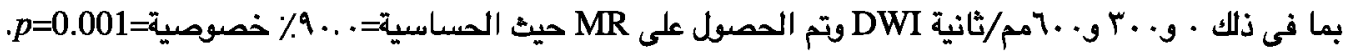

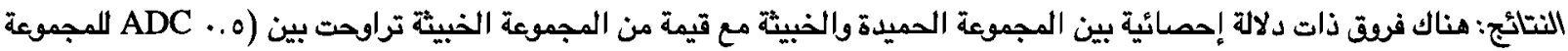

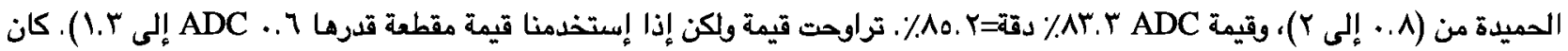

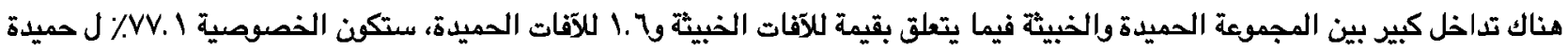

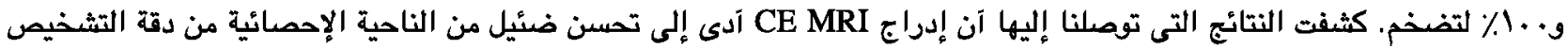

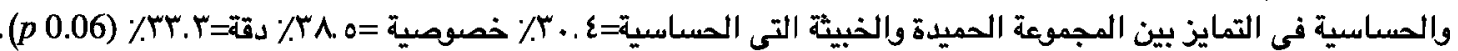

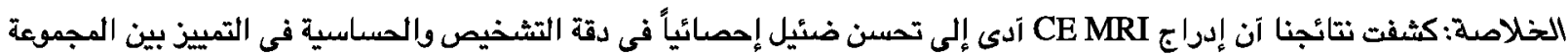

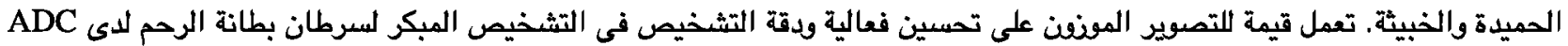
المرضى المعرضين لمخاطر عالية مما يقلل من الحاجة إلى التخخلات الجراحية للتشخيص. 